\title{
Gram-scale, Low-cost and Rapid Fabrication of High-quality Width-controlled One-dimensional Conducting Polymer Nanobelts
}

\author{
Shaojun Guo, Shaojun Dong and Erkang Wang* \\ State Key Laboratory of Electroanalytical Chemistry, Changchun Institute of Applied Chemistry, \\ Chinese Academy of Sciences, Changchun, 130022, Jilin, China, and Graduate School of the \\ Chinese Academy of Sciences, Beijing, 100039, P. R. China
}

\begin{abstract}
Apparatus: TEM measurements were made on a JEOL 2000 transmission electron microscope operated at an accelerating voltage of $200 \mathrm{kV}$. X-ray diffraction (XRD) analysis was carried out on a D/Max 2500 V/PC X-ray diffractometer using Cu (40 kV, $30 \mathrm{~mA})$ radiation. Scanning electron microscopy images were determined with a Philips XL-30 ESEM equipped with energy-dispersive $\mathrm{X}$-ray. The accelerating voltage was $15 \mathrm{kV}$. Transmission infrared spectrum was collected in the transmission mode on a Nicolet 560 FT-IR spectrometer. The Raman instrument includes a FT-Raman spectrometer (Thermo Nicolet 960) equipped with an InGaAs detector and a $\mathrm{Nd} / \mathrm{VO}_{4}$ laser $(1064 \mathrm{~nm})$ as an excitation source. The laser power used was about $380 \mathrm{~mW}$. All spectra are obtained with 512 scans and $4 \mathrm{~cm}^{-1}$ resolution.
\end{abstract}

